\title{
Desenvolvimento de uma abordagem teórica do conceito de ondas térmicas para compreensão da variação de temperatura em filmes finos e sólidos
}

Development of a theoretical approach of the thermals waves concept for understanding of the temperature variation in thin films and solid

\author{
Flávio M. do Couto*1@, Ramon. D. Carvalho², Roberto T. Faria Júnior ${ }^{3}$, Francisco J. M. de \\ Macedo $^{4}$
}

\footnotetext{
${ }^{1}$ Universidade Federal do Espírito Santo, Centro de Ciências Exatas, Naturais e da Saúde, Departamento de Química e Física, Campus de Alegre, Alegre, ES, Brasil.

${ }^{2}$ Universidade Federal de Pelotas, Instituto de Física, Pelotas, RS, Brasil.

${ }^{3}$ Universidade Estadual do Norte Fluminense Darcy Ribeiro, Departamento de Física, Laboratório de Ciências Físicas, Campos dos Goytacazes, RJ, Brasil.

${ }^{4}$ Universidade do Minho, Centro de Física, Braga, Portugal.
}

Recebido em 14 de fevereiro de 2020. Aceito em 24 de fevereiro de 2020.

\begin{abstract}
O presente trabalho estabelece uma análise acerca dos conceitos de ondas eletromagnéticas e sua aplicação no processo de difusão de calor em sólidos com o propósito de compreender e difundir o conceito de ondas térmicas, tão pouco abordado dentro da academia. Com essa análise é possível identificar como a temperatura varia em função das propriedades termofísicas dos materiais quando uma amostra é submetida a uma excitação modulada e periódica por meio de ondas eletromagnéticas. Isso permite a utilização de técnicas fototérmicas, por exemplo, radiometria fototérmica, para determinar as propriedades térmicas de materiais como filmes finos e sólidos homogêneos. O conteúdo aqui desenvolvido pode ser amplamente abordado sob a forma de disciplinas em cursos de graduação e pós-graduação para alunos de física, engenharias e áreas afins.

Palavras-chave: Ondas Térmicas; Ondas Eletromagnéticas; Difusão de Calor em Sólidos; Propriedades Térmicas de Materiais.
\end{abstract}

\begin{abstract}
The current work establishes an analysis of the electromagnetic waves concepts and its application in the process of heat diffusion in solids in order to understand and spread the concept of thermal waves, so little addressed within the academy. It is possible to identify with this analysis how the temperature can change as a function of the thermophysical properties of the materials when a sample is subjected to a modulated and periodic excitation by means of electromagnetic waves. This allows some photothermal techniques, for instance, photothermal radiometry, to determine the thermal properties of materials such as thin films and homogeneous solids. The content developed in this article can be widely addressed in the form of disciplines in undergraduate and graduate courses intended for physics, engineering and students of related fields.
\end{abstract}

Keywords: Thermal Waves; Electromagnetic Waves; Heat Diffusion in Solids.

\section{Introdução}

$\mathrm{O}$ entendimento acerca do conceito físico denominado onda térmica, é subjetivo. Todavia, é fundamental ressaltar que sua natureza, advém da simultaneidade associada ao processo de radiação, como fonte de aquecimento e da propagação de calor e a variação de temperatura, também periódica em seu interior. Nesse sentido, uma amostra sujeita a um aquecimento periódico, a utilização de uma fonte laser, por exemplo, ou seja, modulada ou pulsada, resulta em uma variação de temperatura também periódica na superfície e interior da amostra.

*Endereço de correspondência: fmcfisico@gmail.com
Nesse aspecto a combinação do aquecimento periódico com o processo de difusão de calor na superfície/interior da amostra contribui para a formação de ondas térmicas. Não obstante, a difusão de calor em sólidos não apresenta soluções triviais, todavia existe um grau considerável de complexidade na elaboração de uma teoria que atenda aos parâmetros físicos e matemáticos que a envolve. Sendo assim, algumas soluções possíveis são resultantes de aproximações numéricas, das condições de contorno estabelecidas, das características estruturais, físicas e químicas do material a ser analisado, por exemplo, se são homogêneos e isotrópicos, ou se possuem uma geometria bem definida [1]. A proposta desse artigo é promover uma análise teórica e pormenorizada dos me- 
canismos de transferência de calor, a citar, a radiação eletromagnética e a condução térmica, como processos relevantes para geração de ondas térmicas em amostras sólidas, resultantes das variações de temperatura no interior da amostra e, por conseguinte, definir uma equação para a variação de temperatura em função de algumas propriedades termofísicas do material, dentre as quais, se destacam: a frequência de modulação da fonte de aquecimento; o coeficiente de reflexão fototérmica; a razão entre as efusividades térmicas; o coeficiente e a eficiência de absorção óptica da amostra. Por essa razão, fazer uma descrição dos principais parâmetros envolvidos, tanto na radiação, quanto na condução térmica é fundamental para que se compreenda a geração de ondas térmicas no interior das amostras, tendo em vista que a convecção não contribui de maneira efetiva na geração das mesmas.

\section{Princípios básicos da radiação eletromagnética}

A radiação eletromagnética é formada pela interação simultânea dos campos elétrico e magnético definidos pelas equações (1) e 21), nas quais $\mu$ é a permeabilidade magnética; $\varepsilon$ é a permissividade absoluta e $\sigma$ é a condutividade elétrica da amostra. [2]

$$
\nabla^{2} E=\mu\left(\varepsilon \frac{\partial^{2} E}{\partial t^{2}}+\sigma \frac{\partial E}{\partial t}\right)
$$

$\mathrm{e}$

$$
\nabla^{2} B=\mu\left(\varepsilon \frac{\partial^{2} B}{\partial t^{2}}+\sigma \frac{\partial B}{\partial t}\right)
$$

Se a onda eletromagnética for considerada plana e linearmente polarizada na direção da coordenada $z$, então as equações (1) e 22 tem como soluções as equações (3) e (4). Nas referidas equações as grandezas físicas são: $\omega$ é a frequência angular; $\theta$ é a diferença de fase entre os campos elétrico $E_{(z, t)}$ e o magnético $B_{(z, t)}$, denotados pelas equações (3) e (4) como soluções das equações (1) e (2). Além disso, o parâmetro $k$ representa o número de onda de um material, o qual possui a capacidade de absorver a radiação eletromagnética e convertê-la em calor.

$$
E(z t)=E_{0} e^{[i(k z-\omega t)]}
$$

e

$$
B(z t)=B_{0} e^{[i(k z-j \omega t+\theta)]}
$$

A equação (5) define o número de onda diferenciando da parte real e imaginária, em que

$$
k=k_{r}-i k_{i}
$$

Contudo, as equações (6) e (7) descrevem o comportamento da parte real do número de onda $k_{r}$, assim como da parte imaginária $k_{i}$. Esta, por sua vez, representa a atenuação sofrida pela onda eletromagnética à medida que a profundidade de penetração da radiação aumenta, $\log \mathrm{O}$

$$
\begin{aligned}
& k_{r}=\omega \sqrt{\frac{\varepsilon \mu}{2}}\left[\sqrt{1+\left(\frac{\sigma}{\varepsilon \omega}\right)^{2}}+1\right]^{(1 / 2)} \\
& k_{i}=\omega \sqrt{\frac{\varepsilon \mu}{2}}\left[\sqrt{1+\left(\frac{\sigma}{\varepsilon \omega}\right)^{2}}-1\right]^{(1 / 2)}
\end{aligned}
$$

Nos estudos das propriedades térmicas de materiais é uma das equações base para compreender o processo de aquecimento e geração das ondas térmicas no interior das amostras analisadas. Por exemplo, na determinação das propriedades térmicas de filmes finos e sólidos homogêneos, por meio da radiometria fototérmica modulada no infravermelho [3]. Não obstante, a profundidade de penetração da onda eletromagnética $(\delta)$, definida na equação (8), é inversamente proporcional à atenuação $\left(k_{i}\right)$ equações (7) e (8), sofrida por ela e ao próprio comprimento de onda da radiação incidente, porém diretamente proporcional ao índice de refração imaginário $\left(n_{i}\right)$ definido pela equação $(19)$ [2] [4]

$$
\delta=\frac{1}{k_{i}}=\frac{2 \pi n_{i}}{\lambda}
$$

$$
n=n_{r}-i n_{i}
$$

Dessa forma, o índice de refração do meio também representa uma quantidade complexa definida, assim como o número de onda complexo. O índice de refração está associado à absorção de radiação pelo material. Sendo assim, existem duas soluções para o índice de refração, sendo uma referente a componente real (equação 10) e a outra imaginária (equação 11), logo [4]

$$
n_{r}=c \sqrt{\frac{\varepsilon \mu}{2}}\left[\sqrt{1+\left(\frac{\sigma}{\varepsilon \omega}\right)^{2}}+1\right]^{(1 / 2)}
$$

$$
n_{i}=c \sqrt{\frac{\varepsilon \mu}{2}}\left[\sqrt{1+\left(\frac{\sigma}{\varepsilon \omega}\right)^{2}}-1\right]^{(1 / 2)}
$$

Todavia, se o material em análise não é absorvente, ou seja, se é transparente, o índice de refração imaginário é nulo e a parte real pode ser associada ao número de onda real pela equação reduzida 12

$$
n_{r}=\frac{c k_{r}}{\omega}
$$

Uma vez que, se utilize como fonte de excitação um laser, por exemplo, cuja faixa espectral e de modulação óptica estejam dentro da região do visível, torna-se o suficiente para a determinação dos parâmetros de interesse, associados a uma montagem experimental eficaz. Dentre elas, podem-se destacar a condutividade, a efusividade e a condutividade térmicas de sólidos homogêneos e filmes 
finos [3]. Sendo assim, essas considerações permitem reescrever as equações (6) e (7) e obter como soluções as equações (13) e 14 da seguinte forma

$$
k_{r} \cong k_{i} \cong \sqrt{\frac{\omega \sigma \mu}{2}}
$$

e

$$
\delta=\frac{1}{k_{i}} \cong \sqrt{\frac{2}{\omega \sigma \mu}}
$$

Com base nas equações descritas para o número de onda, mas também da penetração da onda eletromagnética no material, as equações que descrevem os campos elétrico (4) e magnético (5), respectivamente, podem ser reescritas em função da profundidade de penetração da onda eletromagnética, então

$$
E_{(z, t)}=E_{0} e^{(-z / \delta)} \cos \left[\omega t-\left(\frac{z}{\delta}\right)\right] \vec{u}_{x}
$$

e

$$
B_{(z, t)}=B_{0} e^{(-z / \delta)} \cos \left[\omega t-\left(\frac{z}{\delta}\right)-\left(\frac{\pi}{4}\right)\right] \vec{u}_{x}
$$

As equações 15 e 16 atendem às condições consideradas acima para uma onda eletromagnética plana e polarizada e, por essa razão, as tornam soluções gerais para os campos elétrico e magnético. Com isso, é possível determinar o valor médio da energia por unidade de área que chega à superfície da amostra, utilizando o Teorema de Poynting, por meio da equação (17) e do coeficiente de absorção $\left(\beta_{s}\right)$ óptica definido pela equação (18) [2]

$$
S=\frac{1}{2} E_{0}^{2} \frac{k_{r}}{\omega \mu} e^{\left(-\beta_{s} z\right)} \hat{k}
$$

$\mathrm{e}$

$$
\beta_{s}=2 k_{i}=\frac{2 \omega n_{i}}{c}=\frac{4 \pi n_{i}}{\lambda}
$$

Todavia, é pertinente ressaltar algumas considerações acerca das equações e do comportamento das ondas eletromagnéticas, dentre elas a equação (17) permite afirmar que a intensidade da radiação incidente sofre uma atenuação da ordem de $(1 / e)$ para uma profundidade $(\delta)$ e dependerá diretamente das características do material analisado, uma vez que está relacionada ao coeficiente de absorção óptica $\left(\beta_{s}\right)$ da amostra, que por sua vez, é fortemente dependente da frequência da radiação incidente. A partir desse conceito foram desenvolvidas algumas expressões matemáticas que descrevem a interação da radiação com a matéria. Portanto, é comum nesse tipo de interação, considerá-la como um plasma, onde as constantes $\mu=\mu_{0}$ e $\varepsilon=\varepsilon_{0}$ assumem essas equivalências, ou seja, condições de contorno, então o número de onda pode ser reescrito por meio da equação (19) [5] [6]

$$
k=\sqrt{\frac{\left(\omega^{2}-\omega_{p}^{2}\right)}{c^{2}}}
$$

Na equação 19 a grandeza física $\omega_{p}$ - é definida como frequência de plasma e está diretamente relacionada com a interação da radiação com a matéria, sob os seguintes aspectos:

I - Se $\omega_{p}$ for maior que a frequência da radiação, as ondas eletromagnéticas se propagam com atenuação e, por essa razão, o meio se comporta como um condutor metálico absorvente, isso implica em um número de onda complexo.

II - Se $\omega_{p}$ for menor que a frequência da radiação, as ondas eletromagnéticas se propagam sem sofrerem atenuação e, por essa razão, o meio de comporta como um condutor metálico transparente, isso implica um número de onda real.

A frequência de plasma $\omega_{p}$ pode ser quantificada por meio da equação 20, em que e e $m$, são, respectivamente a carga e a massa do elétron, $N$ é o número total de fótons por unidade de volume, enquanto que $\varepsilon_{0}$ é a permissividade elétrica do meio.

$$
\omega_{p}=e \sqrt{\frac{N}{m \varepsilon_{0}}}
$$

Baseado nessas informações, tem se que a interação da radiação com os sólidos pode ocorrer de maneiras distintas, principalmente, porque essas interações estão associadas às características peculiares dos sólidos, por exemplo, a mesma radiação apresentará comportamento diferente se incidir sobre uma amostra opaca, ou transparente [5] [6]. Elas podem ser identificadas como sendo reflexão, absorção, propagação e transmissão e de uma maneira geral podem ser representadas macroscopicamente segundo a Figura 1:

Nesse sentido, definem-se dois parâmetros físicos que são relevantes no estudo de ondas térmicas, porque dependendo do tipo de material eles podem influenciar diretamente na aquisição de dados experimentais e, por conseguinte, trazer informações fundamentais acerca da amostra sólida analisada. Portanto, a Refletância $(R)$ e Transmitância $(T)$ são dois parâmetros relevantes no estudo das propriedades ópticas e térmicas da matéria. Eles são definidos pelas equações 21) e 22), respectivamente, representadas por

$$
R=\frac{J_{r}}{J_{i}}=\frac{S_{r} \cos \theta_{r}}{S_{i} \cos \theta_{i}}=\frac{\left\langle S_{r}\right\rangle}{\left\langle S_{i}\right\rangle}
$$

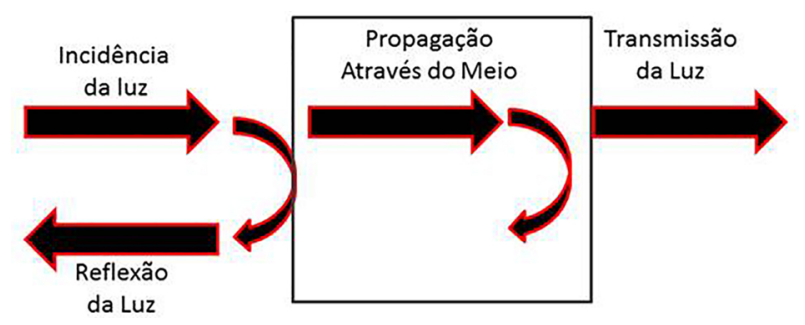

Figura 1: Esquema das diversas formas de interação entre a Luz Incidente (Radiação e/ou Onda Eletromagnética) e um meio sólido: Reflexão, Propagação e Transmissão [5]. 


$$
T=\frac{J_{t}}{J_{i}}=\frac{S_{t} \cos \theta_{t}}{S_{i} \cos \theta_{i}}
$$

Nas equações 21 e 22 o termo físico $\left(J_{i}\right)$ representa a potência da onda eletromagnética que incide sobre um elemento de área infinitesimal e pode ser definida matematicamente por meio da equação (23) [5]

$$
J_{i}=\left|S \hat{n}_{s}\right|=\left|\frac{\varepsilon v E_{0}^{2} \hat{n}_{s}}{2}\right|
$$

em que $v$ é a velocidade de propagação da onda eletromagnética no material e $\hat{n}_{s}$ é o vetor normal à superfície de incidência da radiação. Além disso, $J_{r}$ e $J_{t}$, são respectivamente, a potência refletida e transmitida. Contudo, por conservação da energia pode ser estabelecida a relação $J_{i}=J_{r}+J_{t}$. Utilizando os valores médios dos vetores descritos pela Teoria de Poynting, é possível estabelecer as grandezas físicas $S_{i}, S_{r}$ e $S_{t}$, por meio das seguintes equações (24), (25) e 26) [5]

$$
\begin{aligned}
S_{i} & =\frac{\varepsilon_{i} v_{i} E_{0 i}^{2} \hat{k}}{2}, \\
S_{r} & =\frac{\varepsilon_{r} v_{r} E_{0 r}^{2} \hat{k}}{2}
\end{aligned}
$$

$\mathrm{e}$

$$
S_{t}=\frac{k_{r} E_{0 t}^{2}}{2 \omega \mu} e^{\left(-\beta_{s} z\right)} \hat{k}
$$

Considerando que as frequências de incidência, reflexão, as constantes de permissividade (dielétricas) são iguais (por estarem no mesmo meio) e que a constante de permeabilidade magnética assume o valor de $\mu_{0}$, as equações que descrevem os valores para Refletância $(R)$ e Transmitância $(T)$ podem ser reescritas pelas equações 27 e (28) [5]

$$
R=\left(\frac{E_{0 r}}{E_{0 i}}\right)^{2}
$$

e

$$
T=\left(\frac{E_{0 t}}{E_{0 i}}\right)^{2} \frac{n \cos \theta_{t}}{\cos \theta_{i}}
$$

Um caso particular e de tamanha aplicação experimental consiste em considerar, no processo de alinhamento do feixe incidente, que a incidência sobre a amostra ocorra de forma perpendicular, então as quantidades físicas Refletância e Transmitância podem ser reescritas em termos do índice de refração definidas nas equações (10) e (11), por meio das equações 29 e 30

$$
R=\left|\frac{n-1}{n+1}\right|^{2}=\frac{(n-1)^{2}+n_{1}^{2}}{(n+1)^{2}+n_{1}^{2}}
$$

$$
T=\left|\frac{2 \sqrt{n}}{n+1}\right|=\frac{4 n_{r}+n_{i}^{2}}{\left(n_{r}+1\right)^{2}+n_{i}^{2}}
$$

As equações que determinam os parâmetros físicos descritos acima estão diretamente relacionadas à compreensão das técnicas que utilizam o efeito fototérmico para a determinação de algumas propriedades térmicas, anteriormente descritas, para uma gama de materiais, os quais abrangem os três estados físico da matéria [3]. Não obstante, essas descrições permitem uma compreensão acerca da interação de uma onda eletromagnética com a matéria e, por ventura, permitir determinar alguns parâmetros importantes como a Refletância e Transmitância. Para uma melhor compreensão desse processo, nas próximas seções, far-se-á uma discussão da onda eletromagnética atuando como um gás de fóton e, por conseguinte, no processo de difusão de calor em sólidos, quando estes são submetidos a aquecimentos modulados e periódicos. Portanto, a ideia central é demonstrar a equação da variação de temperatura em sólidos, como resposta às ondas térmicas, resultante do processo de difusão de calor na superfície e no interior dos sólidos, quando submetidos a um aquecimento modulado.

\section{Análise da radiação eletromagnética como um gás de fotóns}

Todo corpo que esteja a uma temperatura diferente do zero absoluto emite ondas eletromagnéticas. Essa energia é liberada sob a forma de radiação, por meio de ondas eletromagnéticas, ou seja, sua faixa espectral de emissão dependerá diretamente da temperatura. Sendo assim, para quantificá-la é necessário o conhecimento de grandezas físicas importantes, por exemplo, o comprimento de onda e a frequência de uma onda eletromagnética [3]. Uma consideração importante é definir a radiação eletromagnética como um gás de fótons. Essa, por sua vez, está em conformidade com as Leis da Estatística de Bose-Einstein, gerando dessa forma uma distribuição de energia, onde o número de estados quânticos ocupados pelos elétrons desse gás de fótons é definido pela equação (31) [7] [8]. Na referida equação, $\varepsilon_{k}$ é a energia do fóton de frequência $\nu$ e $h$ representa a constante de Planck. Além disso, $\mu_{c h}$ (ch-abreviação do inglês chemical) é o potencial químico; $k_{B}$ é a constante de Boltzmann e, por final $\mathrm{T}$ é a temperatura absoluta.

$$
n=\frac{1}{\left\{e^{\left[\left(\varepsilon_{k}-\mu_{c h}\right) / k_{B} T\right]}-1\right\}}
$$

Portanto, se faz necessário estabelecer condições apropriadas para analisar o comportamento desse gás de fótons em uma amostra. Dentre elas, se toda a radiação incidida sobre a sua superfície da amostra for absorvida e emitida na mesma frequência, então ela pode ser tratada como um corpo negro. Além disso, é fundamental ressaltar que estabelecida a condição de equilíbrio termodinâmico para uma dada temperatura, pode-se afirmar que a parcela de energia emitida por unidade de área e tempo independe das características físicas do corpo, sendo apenas dependente da temperatura e da frequência da onda eletromagnética incidente [9] [10] [11]. Nesse sentido, o 
equilíbrio térmico ocorre unicamente por meio da absorção e emissão de fótons pela matéria, em que este número de fótons é variável, uma vez que depende das propriedades físico-químicas de cada material. Sendo assim, a energia livre dos fótons do gás deve ser mínima para uma dada temperatura e volume $(V)$, o que possibilita descrever a equação (32) [11] [12]

$$
\mu_{c h}=\left(\frac{\partial F}{\partial N}\right)_{T, V}=0
$$

Substituindo os valores de $\left(\mu_{c h}=0\right)$ e $\left(\varepsilon_{k}=h \nu\right)$ na equação (31), tem-se a equação (33) denominada Distribuição de Planck

$$
n=\frac{1}{\left\{e^{\left[h \nu / k_{B} T\right]}-1\right\}}
$$

O número de estados quânticos em um dado intervalo de frequência $(v)$ a $(v+d v)$ é determinado pela expressão matemática $\left(8 \pi V v^{2} d v / c^{3}\right)$, em que $c$ é a velocidade de uma onda eletromagnética [7] [8]. Ao multiplicar essa quantidade de estados quânticos pela equação (33) (Distribuição de Planck), considerando o mesmo intervalo de frequência supracitado, é possível determinar o número total de fótons na unidade de volume $V$, conforme a equação 34

$$
d N=\frac{8 \pi V}{c^{3}} \frac{v^{2}}{\left\{e^{\left[h \nu / k_{B} T\right]}-1\right\}} d v
$$

Neste caso, considerando o mesmo intervalo de frequências e o elemento de energia $[d E=(d N / V) h \nu]$, pode-se efetuar a substituição da equação (34) pelo elemento infinitesimal de energia $(d E)$, a fim de obter a equação 35

$$
d E=\frac{8 \pi h}{c^{3}} \frac{v^{3}}{\left[e^{\left(h v / k_{B} T\right)}-1\right]} d v=\frac{8 \pi c h}{\lambda^{5}} \frac{1}{\left[e^{\left(h c / k_{B} T \lambda\right)}-1\right]} d \lambda
$$

A equação (35) proposta por Max Planck define a densidade de radiação, em termos da frequência $(v)$, ou do comprimento de onda $(\lambda)$. Se for adotado como referência a energia irradiada, a partir do espaço semiinfinito por unidade de área e tempo na gama de comprimentos de onda para uma amostra sólida, é possível estabelecer uma relação atemporal por meio da equação (36) [12] [13]. Nesta equação, as constantes possuem valores iguais a $C_{1}=h c / k_{B}=14,388 \mu m K$ e $C_{2}=2 \pi h c^{2}=37,413 W \mu m^{4} / \mathrm{cm}^{2}$.

$$
W^{0}(\lambda, T)=\frac{C_{1}}{\lambda^{5}\left[e^{\left(C_{2} / \lambda T\right)}-1\right]}
$$

Nota-se que a dependência da equação (36) está vinculada ao comprimento de onda e à temperatura [14]. A soma de todas as contribuições de energia $\left[W^{0}(\lambda, T)\right]$ no espectro, em termos do comprimento de onda, se traduz na Lei de Stefan - Boltzmann de acordo com a equação (37), tendo $\sigma_{S B}$ como a constante de Stefan - Boltzmann [14].

$$
W^{0}(T)=\int_{0}^{\infty} W^{0}(\lambda, T)=\sigma_{S B} T^{4}
$$

A energia total é proporcional à quarta potência da temperatura, o que implica em uma variação relevante da energia, quando ocorre uma pequena alteração na temperatura da amostra sólida. A Figura 2 representa um gráfico da densidade de energia radiante de um corpo negro para diferentes temperaturas compreendidas no intervalo entre $300 \mathrm{~K}$ e $1500 \mathrm{~K}$ :

Em relação a equação (36), pode-se afirmar que a exponencial $\left[e^{\left(C_{2} / T \cdot \lambda\right)}>>>1\right]$. Por essa razão, ela pode ser reescrita segundo o modelo conhecido como Limite de Wien em conformidade com a equação (38)

$$
W^{0}(\lambda, T)=\frac{C_{1}}{\lambda^{5}} \frac{1}{e^{\left(C_{2} / \lambda T\right)}}
$$

Quando a análise é feita para grandes comprimentos de onda e/ou temperaturas a equação 38 pode ser reduzida ao aplicar a expansão de Taylor, o que faz com que a exponencial se reduza à quantidade $e^{\left(C_{2} / T \lambda\right)}=$ $C_{2} / \lambda T$. Logo, ela se reduz ao exposto na equação 39 denominada Limite de Rayleigh - Jeans

$$
W^{0}(\lambda, T)=\frac{C_{1}}{\lambda^{5}} \frac{\lambda T}{C_{2}}=\frac{C_{1}}{\lambda^{4}} \frac{T}{C_{2}}
$$

A Figura 3 ilustra a superposição gráfica entre os Limites de Wien, Rayleigh - Jeans e aquela proposta por Planck:

No caso de uma superfície real o espectro da radiação é obtido por meio da equação 40

$$
W(\lambda, T)=\varepsilon(\lambda, T) \cdot W^{0}(\lambda, T)
$$

Nesse perspectiva, a emissividade $[\varepsilon(\lambda, T)]$ é um parâmetro de caracterização das propriedades radiativas da superfície, ou seja, pode ser definida como sendo "a capacidade de um corpo emitir radiação, comparada com a emissão da radiação por um corpo negro à mesma temperatura [12]. Portanto, a emissividade é um parâmetro que depende da parte estrutural do material, mas também do acabamento de sua superfície, podendo variar de acordo com o comprimento de onda, direção de incidência da

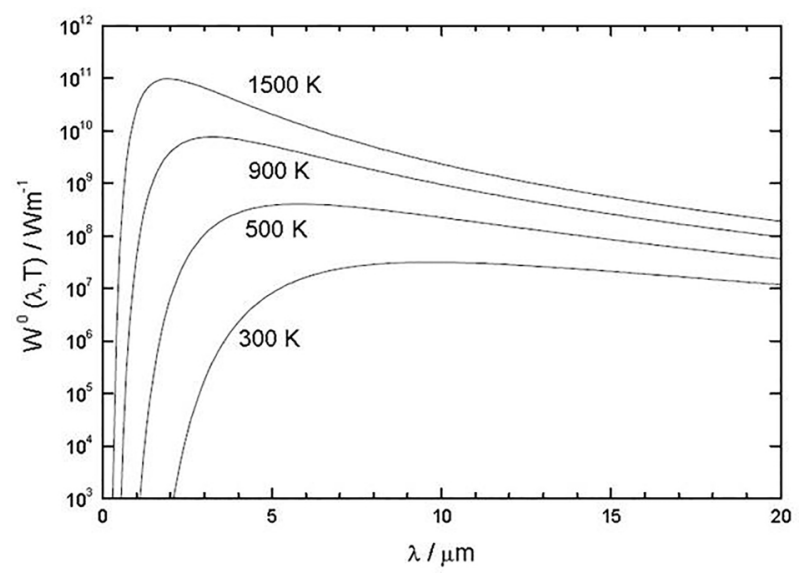

Figura 2: Espectro da Emissão da Radiação de um Corpo Negro para Diferentes Valores de Temperatura [14]. 


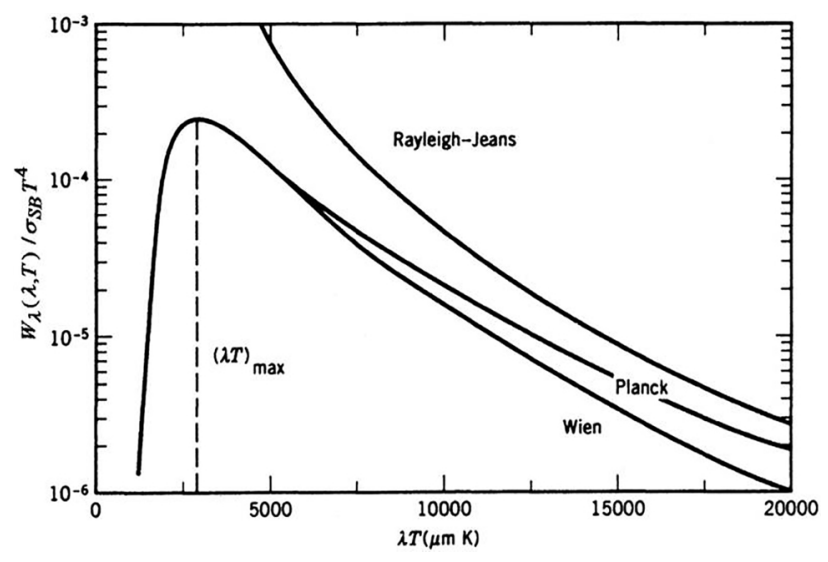

Figura 3: Espectro de Radiação de Energia de um corpo negro hemisférico [14].

radiação e temperatura do material [15]. Um caso especial para os valores da emissividade é atribuído a um tipo específico de material, o qual se comporta como um corpo negro em que $[\varepsilon(\lambda, T)=1]$, enquanto que para os demais materiais $[\varepsilon(\lambda, T)<1][13][14]$.

\section{Ondas térmicas e sua aplicação ao processo de difusão de calor em sólidos}

A difusão de calor em sólidos não apresenta soluções triviais, como já foi descrito na introdução deste artigo, portanto, as soluções apresentadas nesse manuscrito e, posteriormente, a equação para a variação de temperatura em uma amostra, tem como fundamentação e embasamento teórico, as condições de contorno estabelecidas, assim como o atendimento às características específicas dos materiais, a citar, estruturais, físicas e químicas [1]. Nesse sentido, o estudo de ondas térmicas, geradas pela excitação provocada por um feixe de energia modulado/pulsado sobre uma amostra sólida, possibilita a compreensão das propriedades térmicas e ópticas de um material, assim como o fluxo de calor no mesmo. Segundo Carslaw e Jaeger [1], as ondas térmicas geradas em um sólido, devido aos processos microscópicos de excitação, estão de acordo com as equações de difusão de calor, as quais descrevem o comportamento espacial e temporal da distribuição de temperatura no interior da estrutura (amostra) analisada. A Lei de Fourier estabelecida em 1822 afirma, de forma singular, que a taxa de condução de calor (energia térmica) por unidade de área em um sólido pode ser descrita pela equação (41), onde o parâmetro termofísico $k$ representa a condutividade térmica do material, o qual poderá sofrer alterações nos seus valores nas coordenadas espaciais $(x)$ e temporais $(t)$, uma vez que sua dependência está condicionada às composições químicas, físicas e estruturais das amostras submetidas a um aquecimento periódico.

$$
F(x, t)=-k(x, t) \cdot \nabla T(x, t)
$$

O fluxo de calor $F(x, t)$ na equação (41) depende diretamente do gradiente de temperatura $\nabla T(x, t)$, mas também, está associado a equação de difusão de calor em um sólido por meio da equação 42 . Na referida equação $\rho$ é massa específica e c refere-se ao calor específico da amostra sólida, respectivamente.

$$
\rho(x, t) c(x, t) \frac{\partial T(x, t)}{\partial t}=-\nabla F(x, t, T)+Q(x, T)
$$

Sob este aspecto, a amostra se comportará como um meio semi-infinito homogêneo e isotrópico, onde sua superfície estará sujeita à incidência de radiação modulada de frequência $f$, então a intensidade desta radiação pode ser descrita pela seguinte equação (43) [1] [18]

$$
\begin{aligned}
I(x, t) & =\frac{I_{0}}{2}[1+\cos (2 \pi f t)] \rightarrow I(x, t) \\
& =\frac{I_{0}}{2} \operatorname{Re}\left(1+e^{i f t}\right) e^{-\beta_{s} x}
\end{aligned}
$$

A distribuição de calor, fornecido pela fonte, é deduzida da equação proveniente da Lei de Lambert - Beer definida pela equação (34). Nesta equação, o termo físico $\eta_{s}$ é a eficiência de absorção da radiação pelo material, a qual é definida como a razão entre a intensidade total incidente $\left(I_{0}\right)$ em relação àquela convertida e/ou transformada em calor/aquecimento $(I(x, t))$. Não obstante, $\beta$ representa o coeficiente de absorção óptica da amostra (sólida) na faixa espectral do visível. Todavia, tanto $\eta_{s}$, quanto $\beta$ são funções do comprimento de onda da radiação eletromagnética incidente, por essa razão é fundamental fazer uma descrição pormenorizada do mecanismo de transferência de calor, correspondente a radiação eletromagnética, uma vez que, ela desempenha um papel relevante na determinação das propriedades térmicas dos materiais, notoriamente por funcionar como a fonte de aquecimento, a qual permitirá o aquecimento no interior da amostra. Nesse sentido, o fato da radiação incidente ser modulada ou pulsada, provocará um aquecimento periódico no interior da amostra e, por conseguinte, a geração de ondas térmicas.

$$
Q(x, t)=-\eta_{s} \frac{\partial I(x, t)}{\partial x}=R e\left[\frac{1}{2} \eta_{s} \beta_{s} I_{0} e^{-\beta_{s} x} e^{i f t}\right]
$$

A Lei de Lambert - Beer pode ser descrita como sendo: a intensidade da radiação incidente sobre a amostra poderá ser absorvida de maneira parcial, ou total [1] [18]. Mesmo ocorrendo uma absorção parcial da radiação, haverá um aquecimento na superfície da amostra e, por conseguinte, haverá uma difusão de calor para o interior da mesma que dependerá, diretamente, da parcela de radiação absorvida em sua superfície. Desta forma, a quantidade está de acordo com a equação (44) [1] [18]. Considerando que o diâmetro de aquecimento na amostra é maior que o comprimento de difusão térmica $\left(\mu_{t h}\right)$, então, é possível considerar um gradiente de temperatura unidimensional e, por essa razão, reescrever a equação (41), através da 
equação 45 para o processo de difusão térmica [1] [18]

$$
(\rho c)_{(T)} \frac{\partial T(x, t)}{\partial t}=\frac{\partial}{\partial x}\left[k(T) \frac{\partial T(x, t)}{\partial x}\right]+Q(x, T)
$$

Uma amostra sólida ao receber e absorver a radiação proveniente de uma fonte de excitação modulada, em amplitude, apresenta uma equação específica para o processo de difusão de calor da sua superfície para o seu interior, como foi definida pela equação 45 . Esse processo decorre da variação de temperatura $T(x, t)$ (gradiente) na amostra. Essa variação pode ser dividida em duas partes: $T^{d c}(x)$ - representa a parte contínua da temperatura, importando-se apenas com a componente espacial do aquecimento decorrente da absorção da onda eletromagnética por sua superfície e, por conseguinte, sua difusão para o interior da amostra. Entretanto a $T^{t}(x, t)$ referente à parcela variável, uma vez que, tal variação é decorrente das características da fonte de aquecimento (ser modulada ou pulsada), tendo em vista a componente temporal do aquecimento aliada à espacial. É este processo de variação que se torna responsável pela obtenção dos principais sinais fototérmicos de interesse. Ambas as parcelas estão identificadas na equação 46.

$$
T(x, t)=T^{d c}(x)+T^{t}(x, t)
$$

Em termos práticos, tem-se que apenas a parcela variável da temperatura é relevante na definição das equações responsáveis pela geração do sinal. Por essa razão, de acordo com a A Lei de Lambert - Beer o particular interesse está na aferição de $T^{t}(x, t)$ sendo a principal responsável pelas análises de difusão de calor, o que permite desconsiderar a parcela contínua do aquecimento, tendo em vista a sua irrelevância nesse processo [1] [3]. Sendo assim, a equação 46 pode ser reescrita sob o seguinte formalismo apresentado pela equação 47

$$
T_{s}^{t}(x, t)=T(x) e^{i f t}
$$

Portanto, a taxa de variação temporal da temperatura no sólido pode ser definida pela equação 48 como sendo

$$
\frac{\partial T_{s}^{t}\left(x_{s}, t\right)}{\partial t}=\left[\alpha_{s} \frac{\partial^{2} T^{t}\left(x_{s}, t\right)}{\partial x_{s}^{2}}\right]+\frac{\eta \beta_{s} I_{0}}{2(\rho c)_{s}} e^{-\beta_{s} x} \operatorname{Re}\left\{e^{i f t}\right\}
$$

Uma solução viável da equação 48 para o desenvolvimento do modelo teórico empregado na difusão de calor em amostras sólidas, foi estabelecida por Carslaw e Jaeger [1] e Bein e colaboradores [6], segundo as equações 49 e (50), em que os sub índices (s) e (g) referem-se às contribuições para a variação de temperatura na amostra sólida e no gás adjacente à superfície do material, então

$$
T_{s}^{t}(x, t)=\left[A_{s} e^{\sigma_{s} x}+B_{s} e^{-\sigma_{s} x}+C_{s} e^{-\beta_{s} x}\right] e^{i f t}
$$

e

$$
T_{g}^{t}(x, t)=\left[A_{g} e^{\sigma_{g} x}+B_{g} e^{-\sigma_{g} x}\right] e^{i f t}
$$

A Figura 4 ilustra um esquema geométrico arbitrário das regiões descritas acima com as respectivas grandezas

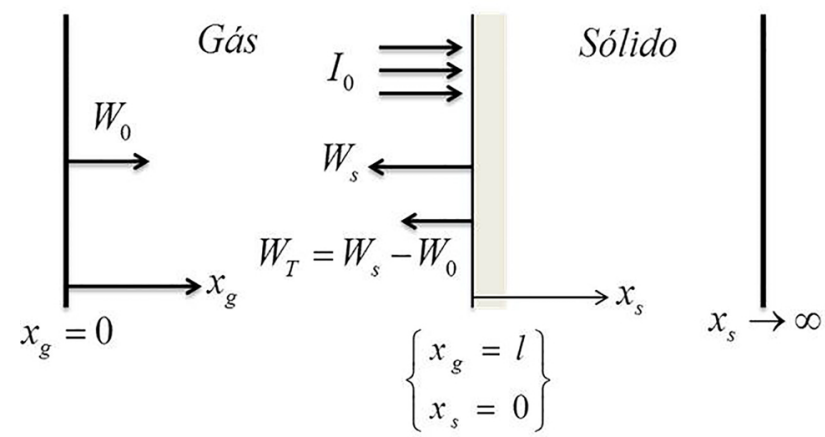

Figura 4: Esquema Geométrico da Incidência de Radiação em Sólido em Contato com uma Região de Gás [10].

físicas envolvidas, como as notações do fluxo de calor $(W)$ proveniente da fonte e da amostra, assim como a incidência da Radiação $\left(I_{0}\right)$ e os possíveis limites espaciais $(x)$, os quais delimitam as regiões fronteiriças entre os meios sólido e gasoso:

De um modo geral, a acepção teórica dos conceitos envolvidos nos estudos das ondas térmicas aplicado aos estudos da variação de temperatura em sólidos, é comum considerar apenas as contribuições da radiação (fonte) e condução (amostra), uma vez que as mudanças de temperatura ocorridas na interface gás - sólido, não expressam de maneira relevante uma corrente de convecção, capaz de atribuir uma parcela quantitativa aos sinais da amplitude e fase dos sinais medidos, tão pouco na aquisição dos dados para fins de estudos [17] [18]. Por essa razão, torna-se primordial estabelecer algumas condições de contorno para tratar o caso especial da transferência de calor por radiação/condução, visualizada no modelo esquemático da Figura 4, onde se destacam [10] [18] [19]:

I - Nas superfícies, $\left(x_{g}=0\right)$, a variação de temperatura assume valores bem definidos nas equações (51), (52) e (53):

$$
T_{g}^{t}(0, t)=T_{s}^{t}\left(l_{s}, t\right)=0
$$

II - Na interface gás / sólido considerar-se-á uma temperatura constante, logo:

$$
x_{g}=l, \quad x_{s}=0 \rightarrow T_{g}^{t}\left(l_{g}, t\right)=T_{s}^{t}(0, t)
$$

III - O Fluxo de Calor contínuo na interface gás/sólido será obtido levando em consideração a seguinte igualdade:

$$
k_{g} \frac{\partial T_{g}^{t}\left(x_{g}, t\right)}{\partial x_{g}}\left|x_{g}=l_{g}=k_{s} \frac{\partial T_{s}^{t}\left(x_{s}, t\right)}{\partial x_{s}}\right| x_{s}=0
$$

Segundo Almond e Patel [16], há soluções previstas para as equações 49 e (50), desde que considere a componente periódica da solução. Nestas mesmas equações o termo $\sigma$, denominado vetor de onda complexo, é definido por meio da equação (54). Nesta equação $\alpha$ representa a difusividade térmica, definida como a capacidade que uma amostra sólida tem de difundir o calor (energia) e $\mu_{t h}$ (th - abreviação do inglês thermal) é o comprimento de difusão térmica, compreendido como a profundidade 
máxima de penetração de uma onda térmica, consonante ao processo de difusão de calor em seu interior, ou seja, fisicamente estabelece a atenuação com que a amplitude da onda térmica é fortemente amortecida no interior da amostra.

$$
\sigma=(1+i) \sqrt{\pi f / \alpha}
$$

Os parâmetros descritos no parágrafo anterior podem ser calculados por meio das equações 55 e (56). É fundamental destacar que essas propriedades dependem diretamente das composições químicas de cada amostra, mas também do processo de aquecimento pelo qual elas foram submetidas, ou seja, do tipo de radiação eletromagnética (frequência, modulada ou pulsada, intensidade) $[16]$.

$$
\alpha=\frac{k}{\rho c}
$$

$\mathrm{e}$

$$
\mu_{t h}=\sqrt{\alpha / \pi f}
$$

Nesse sentido é relevante ressaltar que, quanto maior a frequência de modulação, menor será a penetração da onda térmica no interior da amostra, por ser inversamente proporcional à frequência de modulação do feixe incidente. Além disso, os parâmetros supracitados estão diretamente relacionados com outras propriedades térmicas de interesses acadêmicos e tecnológicos, a citar, $k$, já descrito como a condutividade térmica na equação (41) $(k)$, compreendida como a capacidade de conduzir o calor pela amostra; $\rho c$ é a capacidade térmica calorífica, definida pela eficácia em armazenar o calor em uma amostra. Contudo, é possível atrelar a esses parâmetros termofísicos, outro de suma importância dentre as propriedades térmicas gerais, denominada efusividade térmica, também conhecida como inércia térmica, a qual pode ser calculada via equação (57) [3] [10]

$$
e=\sqrt{(k \rho c)_{s}}
$$

No entanto, nas situações de baixas frequências e grandes difusividades térmicas, a radiação, sob a forma de onda térmica, penetrará consideravelmente na amostra. Sendo assim, haverá uma gama de informações provenientes da amostra por conta desse formalismo [3] [10]. No caso particular em que $\left(x_{t h}=\mu_{t h}=\sqrt{\alpha / \pi f}\right)$, a radiação decai de $(1 / e)$ do seu valor inicial, sendo $e$ o número de Euler [3] [18] [19].

Contudo, uma vez definidos os parâmetros térmicos que podem ser mensurados por meio das equações (55), (56) e (57), é crucial definir os termos $(A)$ e $(B)$ presentes as equações (49) e (50). Tendo em vista a gama de condições estabelecidas nos parágrafos anteriores, no que diz respeito ao comportamento das ondas térmicas nas interfaces gás/ sólido, das condições de continuidade do fluxo modulado de calor na amostra em consonância ao processo de difusão de calor, além das considerações feitas acerca da temperatura, é possível concluir que o parâmetro $\left(A_{s}=0\right)$, por conta das condições de contorno definidas pelas equações $(51),(52)$ e 53 e o termo $\left(B_{s}\right)$, é calculado por meio da equação (58) [10]

$$
\begin{aligned}
& B_{s}=-C_{s} \frac{\beta_{s}}{\sigma_{s}} \frac{\left[1+(R+g) \frac{\sigma_{s}}{\beta_{s}}\right]}{[1+R+g]} \\
& \Rightarrow\left\{\begin{array}{c}
R=\frac{4 \varepsilon_{s} \sigma_{S B} T_{s}^{3}(0)}{k_{s} \sigma_{s}}=|R|=\frac{2 \sqrt{2} \varepsilon_{s} \sigma_{S B} T_{s}^{3}(0)}{\sqrt{\pi \omega} \sqrt{(k \rho c)_{s}}} \\
g=\frac{k_{g} \sigma_{g}}{k_{s} \sigma_{s} \tanh \left(\sigma_{g} l_{g}\right)} \rightarrow \tanh \left(\sigma_{g} l_{g}\right) \cong 1 \\
\text { então } \rightarrow g=\frac{\sqrt{(k \rho c)_{g}}}{\sqrt{(k \rho c)_{s}}}
\end{array}\right.
\end{aligned}
$$

A constante $\left(C_{s}\right)$ refere-se a parcela não homogênea do sólido e é obtida por meio da equação (69)

$$
C_{s}=-\frac{\eta_{s} I_{0}}{2 k_{s} \beta_{s}\left[1-\left(\frac{\sigma_{s}}{\beta_{s}}\right)^{2}\right]}
$$

Finalmente, a solução complexa da equação (59), dependente da frequência de modulação da radiação incidente na superfície da amostra, capaz de promover o aquecimento periódico e, consequentemente, a difusão de calor. Portanto se $\left(x_{s}=0\right)$, a equação (69) pode ser reescrita na forma da equação (60), em que $g$ é definido como a razão entre as efusividades térmicas da amostra e do meio adjacente a sua superfície, sendo um parâmetro termofísico relevante na determinação das propriedades térmicas de filmes finos e sólidos homogêneos [3] [10] [16] [17].

$$
\begin{aligned}
& T_{s}^{t}\left(x_{s}, t\right)=\frac{\eta_{s} I_{0}}{2} e_{s} \sqrt{2 \pi f}\left[1-\left(\frac{\sigma_{s}}{\beta_{s}}\right)^{2}\right] \\
& \times\left[\frac{\left[1+(R+g) \frac{\sigma_{s}}{\beta_{s}}\right]}{[1+(R+g)]}-\frac{\sigma_{s}}{\beta_{s}}\right] e^{[i(2 \pi f t-\pi / 4)]}
\end{aligned}
$$

Nesse contexto, pode-se destacar o coeficiente de reflexão térmica $(R)$, sendo um número complexo que representa a perda de calor por radiação na interface gás/sólido, sendo ele um parâmetro dependente da temperatura da amostra, das propriedades ópticas do material e da frequência de modulação [18]. Portanto, embora o conceito de ondas térmicas, seja um tanto, quanto subjetivo, a equação (60) que explicita a variação de temperatura no espaçotempo no interior de uma amostra sólida, é resultante de um aquecimento periódico em sua superfície e, posterior, periodicidade do transporte de energia para seu interior, ou seja, uma onda térmica.

\section{Conclusão}

Os conceitos de ondas térmicas elucidados nesse artigo podem ser desenvolvidos em uma disciplina específica, com alunos dos cursos de graduação e pós-graduação, pertencentes às áreas das Ciências Exatas, principalmente, por ter sido fundamentado em uma análise, pormenorizada, do comportamento de ondas eletromagnéticas 
e propagação de calor em sólidos, quando submetidos à excitação/aquecimento por uma fonte modulada/pulsada. Isso permitiu uma melhor compreensão acerca da variação de temperatura em uma amostra sólida, mas também a notória aplicação desse conceito na determinação de diversas propriedades térmicas, por meio de técnicas Fototérmicas [3] [16] [17] [19] [20]. Portanto, a equação 60, em outras palavras, define o aquecimento periódico da amostra dependente da variação de temperatura na amostra no espaço/tempo, por essa razão, ela pode ser interpretada como uma onda térmica [20] [21]. Além disso, é fundamental ressaltar que a os valores da temperatura na amostra estão diretamente relacionados aos parâmetros termofísicos descritos nos parágrafos supracitados.

\section{Agradecimentos}

À Coordenação de Aperfeiçoamento de Pessoal de Nível Superior - CAPES

À Fundação de Amparo à Pesquisa e Inovação do Espírito Santo - FAPES

\section{Referências}

[1] H.S. Carslaw e L.C. Jaeger, Conduction of Heat in Solids (Oxford University Press, Clarendon, 1959), 2 ${ }^{\mathrm{a}}$ ed., v. 1.

[2] D.J. Griffiths, Introduction to Electrodynamics (Prentice Hall, Englewood Cliffs, 1989), $2^{\mathrm{a}}$ ed.

[3] F.M. Couto, Caracterização das Propriedades Térmicas de Filmes Finos e Sólidos Homogêneos por Radiometria Fototérmica Modulada no Infravermelho. Tese de Doutorado, Universidade Estadual do Norte Fluminense Darcy Ribeiro, Rio de Janeiro (2015).

[4] F.L. Pedrotti e S.J.L. Pedrotti, Introduction to Optics (Prentice Hall, Upper Saddle River, 1996), $2^{\mathrm{a}}$ ed.

[5] M. Fox, Optical properties of solids (Oxford University Press, New York, 2003).

[6] B.K. Bein e J. Pelzl, in: Plasma Diagnostics, Surface Analysis and Interactions, editado por O. Auciello e D.L. Flamm (Cambridge University Press, Cambridge, 1989), v. 2 , p. 211.

[7] A.J. Pointon, Introduction to Statistical Physics (Longmans Green and Co., Londres, 1967).

[8] L.D. Landau e E.M. Lifshitz, Statistical Physics (Butterworth-Heinemann, Oxford, 1968).

[9] S.R. Dahmen, Revista Brasileira de Ensino de Física 27, 271 (2005).

[10] A. Haj-Daoud, Photothermal Materials Characterization at Higher Temperature by Means of IR Radiometry. Masters Dissertation, Zur Erlangung des Grades eines Doktors der Naturwissenschaften der Fakultat fur Physik und Astronomie van der Ruhr-Universitat, Bochum (1999).

[11] W. Nolting, GrundKurse Theoretische Physik 6 (Zimmermann Neufang, Ulmen, 1994).

[12] R. Siegel e J.R. Howel, Thermal radiation Heat Transfer (McGraw-Hill Book Company, New York, 1981).

[13] R.D. Hudson, Infrared System Engineering (Wiley, New York, 1969).
[14] M.Q. Brewster, Thermal radiative transfer and properties (John Wiley and Sons, New York, 1992).

[15] E.M. Sparrow e R.D. Cess, Radiation Heat Transfer (Mcgraw - Hill Book Company, Washington, 1978).

[16] F. Macedo, F. Vaz, L. Rebouta, P. Carvalho, A. HajDaoud, K.H. Junge, J. Pelzl e B.K. Bein, Vacuum 82, 1457 (2008).

[17] F. Macedo, A. Gören, F. Vaz, J.L. Nzodoum Fotsing, J. Gibkes e B.K. Bein, em: Fhotoacustic $\& 3$ Photothermal Phenomena editado por F. Scudieri a. M. Bertolotti (EDP Sciences, Rio de Janeiro, 2005).

[18] A. Rosencwaig, J. Opsal, W.L. Smith e D.L. Willenborg, Appl. Phys. Lett. 46, 1013 (1985).

[19] C.A. Bennett e R.R. Patty, Applied Optical 21, 49 (1982).

[20] D.P. Almond e P.M. Patel, Photothermal Science and techniques (Chapman \& Hall, London, 1996).

[21] A. Salazar e A. Sánchez-Lavega, Rev. Sci. Instrum. 65, 2896 (1994). 